\title{
New winds in GPCR-based drug discovery
}

\author{
"The recent substantial technology development and success in \\ G-protein-coupled receptor crystallography has further opened new \\ avenues for rational design."
}

\begin{abstract}
Keywords: allosteric modulations $\bullet$ binding technologies $\bullet$ GPCR structures - ligand screening $\bullet$ virtual screening
\end{abstract}

First draft submitted: 14 January 2016; Accepted for publication: 18 January 2016; Published online: 22 April 2016

\section{"Structure-based approaches are highly impactful on GPCR drug development"}

- Kenneth Lundstrom

G-protein-coupled receptors (GPCRs) represent the largest family of drug targets for a wide variety of indications including cardiovascular, metabolic, neurodegenerative, infectious and oncologic diseases [1,2]. More than 800 human GPCRs mediate signal transduction events triggered by hormones, neurotransmitters, ions, peptides, amino acids, odors, taste and light $[1,3]$. Disruption in GPCR function leading them to become inactive, overexpressed or constitutively active can cause alterations in ligand binding, coupling to $\mathrm{G}$ proteins, receptor desensitization and recycling resulting in the potential onset of disease [4]. In addition to coupling to $G$ proteins, GPCRs also signal through G-protein-independent GPCR kinases and $\beta$-arrestins [5], which has added a new dimension to GPCR-based drug discovery. Moreover, GPCR dimerization has been demonstrated for several receptors affecting the pharmacology and signaling properties [6].

Traditionally, GPCRs have been screened for lead compounds using receptor-binding assays [7]. To replace utilization of radioactive ligands, time-resolved fluorescence resonance energy transfer has provided the means to label GPCRs expressed on the cell surface [8]. Although useful for receptor screening, binding assays present limitations due to the lack of appropriate cell lines expressing tagged GPCRs and fluorophore-labeled ligands. Moreover, although agonists and antagonists can be characterized in the same binding assay, it does not allow differentiation between agonists, antagonists and inverse agonists among candidate compounds. Therefore, a shift toward functional assays has been evident as it provides better possibilities to analyze the action of compounds and their effect as potential novel drug candidates.

A number of functional assays have been established for GPCRs [3], originally based on measurement of intracellular $\mathrm{Ca}^{2+}$ release by a Fura-2 assay [9]. Moreover, fluorescent dyes such as Fluo-3 and Fluo-4 and the automated fluorescence plate reader FLIPRTM (Molecular Devices, CA, USA) has allowed automation and ultra-high-throughput screening capacity for GPCRs in 384 and 1536 formats [10]. The nonradioactive method for inositol 1, 4, 5-triphosphate $\left(\mathrm{IP}_{3}\right)$ accumulation named IP-One

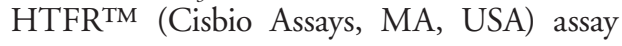
measures reduction in energy transfer between acceptor IP and a europium-conjugated IP1 antibody and has been applied for highthroughput screening in 1536 format for M1 acetylcholine, vasopressin V1b and neuropeptide $S$ receptors [11]. Other functional assays for GPCRs include GTP $\gamma S$ binding [12] and cAMP stimulation [13]. Engineering of reporter gene assays has also contributed strongly to drug discovery on GPCRs. In this context, elements within promoter regions such as the cAMP response element, the nuclear factor of activated T-cell response element and the serum response element have been utilized [14]. Luciferase, alkaline phosphatase, $\beta$-galactosidase and $\beta$-lactamase expression has been applied for colorimetric readouts, fluorescence or

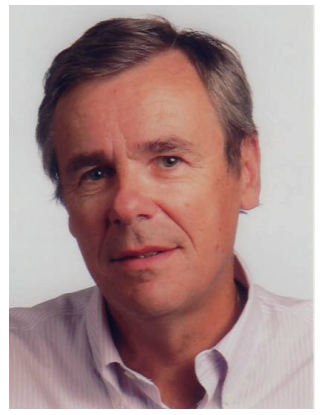

Kenneth Lundstrom

PanTherapeutics, Rue des Remparts 4, CH1095 Lutry, Switzerland Tel. +41-79 7766351

lundstromkenneth@gmail.com 
luminescence at 1536 and 3456 formats [15]. Application of quenching resonance energy transfer has been used for time-resolved fluorescence cAMP screening of $\beta 2$-adrenergic and $\delta$-opioid receptors in HEK293 cells, which demonstrated agonist-mediated stimulation of GPCRs coupled to either stimulatory $\left(\mathrm{G}_{\mathrm{s}}\right)$ or inhibitory $\left(G_{i}\right) G$ proteins [16]. In another approach, two inactive $\beta$-lactamase fragments (bla[a] and bla[b]) where fused to the $\beta 2$-adrenergic receptor ( $\beta 2 \mathrm{AR}$ ) and $\beta$-arrestin, respectively, in a reporter assay in stable HEK293 cells for measurement of cAMP stimulation [17]. Dose-dependent responses to $\beta 2 \mathrm{AR}$ agonists and antagonists were observed and $\beta 2 A R$ agonists were identified from a natural compound library in a high-throughput screening setting.

G-protein-independent signaling of GPCRs might provide alternative opportunities for development of novel drugs. In this context, high-resolution fluorescence microscopy and automated image analysis have been applied for the quantification of GPCR internalization [18]. The multidimensional readouts and the determination of temporal and spatial features of targets are clear assets of this approach. Although specific fluorophore-labeled ligands and antibodies against extracellular receptor domains or $\mathrm{N}$-terminal epitope tags have been applied, GFP- or RFP- tagged GPCRs have been preferentially used for large-scale drug screening programs [19]. Furthermore, fluorescence-based assays have been developed for GFP-tagged $\beta$-arrestin which allow visualization of $\beta$-arrestin localization and GPCR internalization. In the context of non-imaging-based $\beta$-arrestin assays, bioluminescence resonance energy transfer has been applied for high-throughput screening [20] and, for example, when GPCRs have been tagged with GFP or YFP tags at the C-terminus and $\beta$-arrestin with a Renilla luciferase, light emission for the proximity of Renilla luciferase and GFP allows detection of a higher wavelength [21]. Alternatively, when $\beta$-arrestin was fused to a tobacco etch virus (TEV) protease cleavage site and the transcription factor Gal-VP16 to the C-terminus of a GPCR, the protease-tagged $\beta$-arrestin interacted with the GPCR and cleaved off Gal-VP16, which was transported to the nucleus and induced $\beta$-lactamase reporter transcription [22]. In another approach, $\beta$-arrestin was fused to $\beta$-galactosidase with a deletion in the $\mathrm{N}$-terminus and the $\mathrm{N}$-terminal deletion of $\beta$-galactosidase was fused to the C-terminus of the GPCR of interest. Interaction of $\beta$-arrestin with the GPCR brings the two $\beta$-galactosidase fragments together resulting in measurable $\beta$-galactosidase chemiluminescence [23]. Furthermore, bimolecular fluorescence complementation applied to GPCRs and $\beta$-arrestin provides the means for a measurable signal once the receptor and $\beta$-arrestin interact [24]. Moreover, the PathHunter $\beta$-arrestin recruitment technology (DiscoverX, Freemont, CA, USA) was utilized for screening of agonists to the human endothelial differentiation sphingolipid GPCR1 (EDG1) [25]. The outcome was 2157 agonist hits from 345,052 compounds tested of which only ten showed $\beta$-arrestin recruitment activity of a nonrelated GPCR. The importance of signaling through $\beta$-arrestins for therapeutic activity as antipsychotic and antimanic agents was evaluated in a study of unique dopaminergic D2 receptor agonists [26]. Unprecedented $\beta$-arrestin-biased ligands for Gi-coupled GPCRs were discovered and they acted as partial agonists for dopaminergic D2 receptor- $\beta$-arrestin interaction displaying potent antipsychotic-like activity in C57BL/6 mice.

Application of bioinformatics, genomics and in silico technologies has generated data on GPCRs for which no ligands are known. Much attention has been to deorphanize these orphan GPCRs in attempts to discover novel drug targets [27]. One approach has been to analyze functional activity of RAIG1, GPRC5B and GPRC5C when fused to the N-terminal domain of the homologous metabotropic glutamate receptor 1 [28]. $\mathrm{Ca}^{2+}$ mobilization assays have been used in high-throughput format for orphan receptors [29]. In the case of cytotoxic GPCRs, such as the stress-inducible antiproliferative cell cycle regulator G2A, a functional assay was established based on the inducible T-Rex expression system with $\beta$-lactamase readout [30], which allowed the identification of G2A agonists from a chemical library containing 740,000 compounds. Additionally, a GTP $\gamma S$ scintillation proximity assay has been developed in a 1536well format for orphan GPCR screening [31]. In a systematic investigation, the human 'GPCR-ome', which included more than 150 orphan receptors was examined for emerging trends in GPCR drug discovery [32]. In another approach related to deorphanization of GPCRs involving peptidomics, MS has been applied for highthroughput characterization of endogenous peptides in the nervous and endocrine systems for the discovery of potential drug targets [33].

The discovery that GPCR dimerization affects receptor pharmacology and signaling has added a new dimension to drug development [34]. Certain class C GPCRs require heterodimerization to be functionally active while many class A GPCRs have been discovered to exist as homodimers. For instance, the Gai-coupled cannabinoid-1 receptor (CB1R) and the Gaq-coupled angiotensin-1 receptor (AT1R) showed potentiation of AT1R signaling and coupling of AT1R to multiple G proteins. AT1R-CB1R heterodimers have been demonstrated to provide 'heterodimer-directed signaling specificity', which potentially modifies the physiological role of GPCRs in a disease-specific regulation [35]. Dimerization assays are based on fluorescence resonance energy 
transfer (FRET) and bioluminescence resonance energy transfer (BRET) with donor and acceptor molecules engineered at the C-termini of respective GPCRs [36,37]. A combination of time-resolved fluorescence resonance energy transfer and SNAP-tag (mutant of DNA repair protein $0^{6}$-alkylguanine DNA alkyltransferase) allows analysis of protein-protein interaction on the surface of living cells in 96- and 384-well formats [38].

In silico modeling and structure-based approaches have become an important part of drug discovery and development today [39]. For instance, in silico modeling using multiple templates from the extracellular loops of the orphan GPR17 was applied to identify potential ligands for neurodegenerative diseases [40]. Virtual screening of the GPR17 binding site was supported by functional and pharmacological validation, which led to the identification of five agonists and partial agonists with diverse chemical structures. As x-ray structures are available for 20 GPCRs and more than 60 ligands, new details of GPCR-ligand interaction such as ligand binding modes outside the orthosteric pocket have significantly affected the drug discovery process [41]. Highresolution structures have enabled docking and virtual screening of GPCRs and furthermore have allowed refinement and post-processing to improve the accuracy of calculations [42]. These approaches have cast some light on the mechanisms of receptor activation and have revealed novel ligand-binding sites [43], which has provided new possibilities for rational drug design. As GPCRs are allosteric proteins where their signal transduction relies on interactions between topographically distinct domains structure, determination of GPCRs has also aided in targeting allosteric sites in the context of new drug development [44].

In summary, progress in technology development including miniaturization and automation has accelerated drug discovery in general and also for GPCRs.

\section{References}

1 Bockaert J, Pin JP. Molecular tinkering of G protein-coupled receptors: an evolutionary success. EMBO J. 18(7), 1723-1729 (1999).

2 Thompson MD, Burnham WM, Cole DE. The G proteincoupled receptors: pharmacogenetics and disease. Crit. Rev. Clin. Lab. Sci. 42(4), 311-392 (2005).

3 Lundstrom K. Present and future approaches to screening of G protein-coupled receptors. Future Med. Chem. 5(5), 523-538 (2013).

4 Thompson MD, Percy ME, McIntyre Burnham W et al. G protein-coupled receptors disrupted in human genetic disease. Methods Mol. Biol. 448, 109-137 (2008).

5 Lutrell LM, Lefkowitz RJ. The role of beta-arrestins in the termination and transduction of G-protein-coupled receptor signals. J. Cell Sci. 115(3), 455-465 (2002).
However, the number of novel drugs reaching the market has been disappointingly low. The stricter safety requirements, poor target validation and difficulties of translation to clinic have certainly contributed to the modest success rate. Additionally, the high lipophilic content of high-throughput screening compounds as well as economic factors such as reimbursement policies has negatively affected the outcome. Is there any hope of an upswing in GPCR drug discovery? Although there might be some suggestions of a 'saturation' of GPCR screening capacities, it needs to be pointed out that only some 60 of the total 800 GPCRs have been subjected to drug development. Moreover, only a small proportion of the available 'chemistry space' has been explored so far. Furthermore, functional coupling to $G$ proteins and G-protein-independent GPCR functions have provided new drug discovery opportunities. The unique properties of GPCR dimers might generate possibilities for finding more potent drugs. Allosteric modulations of GPCRs have also contributed to the discovery of new medicines. The recent substantial technology development and success in GPCR crystallography has further opened new avenues for rational design. Overall, it seems that there are indeed some new favorable winds in the field of GPCR drug development.

\section{Financial \& competing interests disclosure}

The author has no relevant affiliations or financial involvement with any organization or entity with a financial interest in or financial conflict with the subject matter or materials discussed in the manuscript. This includes employment, consultancies, honoraria, stock ownership or options, expert testimony, grants or patents received or pending, or royalties.

No writing assistance was utilized in the production of this manuscript.

6 Rozenfeld R, Devi LA. Exploring a role for heteromerization in GPCR signaling specificity. Biochem. J. 433(1), 11-18 (2011).

7 Franchet C, Dorange I. GPCR binding technologies: an overview. Curr. Top. Med. Chem. 15(24), 2476-2483 (2015).

8 Degorce F, Card A, Soh S et al. HTRF: a technology tailored for drug discovery - a review of theoretical aspects and recent applications. Curr. Chem. Genomics 3, 22-32 (2009).

9 Eistetter HR, Mills A, Brewster R et al. Functional characterization of neurokinin-1 receptors on human U373MG astrocytoma cells. Glia 6(2), 89-95 (1992).

10 Zhang Y, Kowal D, Kramer A et al. Evaluation of FLIPR calcium 3 assay kit - a new no-wash fluorescence calcium indicator reagent. J. Biomol. Screen. 8(5), 571-577 (2003).

11 Liu K, Titus S, Southall N et al. Comparison on functional assays for Gq-coupled GPCRs by measuring inositol monophosphate-1 and intracellular calcium in 1536-well plate format. Curr. Chem. Genomics 1, 70-78 (2008). 
12 Labrecque J, Wong RS, Fricker SP. A time-resolved fluorescent lanthanide (Eu)-GTP binding assay for chemokine receptors as targets in drug discovery. Methods Mol. Biol. 552, 153-169 (2009).

13 Williams C. cAMP detection methods in HTS: selecting the best from the rest. Nat. Rev. Drug Discov. 3(2), 125-135 (2004).

14 Cheng Z, Garvin D, Paguio A et al. Luciferase reporter assay system for deciphering GPCR pathways. Curr. Chem. Genomics 4, 84-91 (2010).

15 Fan F, Wood KV. Bioluminescent assays for high-throughput screening. Assay Drug Dev. Technol. 5(1), 127-136 (2007).

16 Martikkala E, Rozwandowicz-Jansen A, Hänninen P et al. A homogenous single-label time-resolved cAMP assay. J. Biomol. Screen. 16(3), 356-362 (2011).

17 Zhao CK, Yin Q, Li SY. A high throughput screening system for $\mathrm{G}$ protein-coupled receptors using $\beta$-lactamase enzyme complementation technology. Acta Pharmacol. Sin. 31(12), 1618-1624 (2010).

18 Zanella F, Lorens JB, Link W. High content screening: seeing is believing. Trends Biotechnol. 28(5), 237-245 (2010).

19 Hirasawa A, Tsumaya K, Awaji T et al. Free fatty acids regulate gut incretin glucagon-like peptide-1 secretion through GPR120. Nat. Med. 11 (1), 90-94 (2005).

20 Hamdan FF, Audet M, Garneau P et al. High-throughput screening of $\mathrm{G}$ protein-coupled receptor antagonists using a bioluminescence resonance energy transferl-based betaarrestin 2 recruitment assay. J. Biomol. Screen. 10(5), 463-475 (2005).

21 Kamal M, Marquez M, Vauthier V et al. Improved donor/ acceptor BRET couples for monitoring beta-arrestin recruitment to G protein-coupled receptors. Biotechnol. J. 4(9), 1337-1344 (2009).

22 Barnea G, Strapps W, Herrada G et al. The genetic design of signaling cascades to record receptor activation. Proc. Natl Acad. Sci. USA 105(1), 64-69 (2008).

23 Zhao X, Jones A, Olson KR et al. A homogenous enzyme fragment complementation-based beta-arrestin translocation assay for high-throughput screening of $\mathrm{G}$ protein-coupled receptors. J. Biomol. Screen. 13(8), 737-747 (2008).

24 Kilpatrick LE, Holliday ND. Disecting the pharmacology of G protein-coupled receptor signaling complexes using bimolecular fluorescence complementation. Methods Mol. Biol. 897, 109-138 (2012).

25 van der Lee MM, Bras M, van Koppen CJ et al. Betaarrestin recruitment assay for identification of agonists for the sphingosine-1 phosphate receptor EDG1. J. Biomol. Screen. 13(10), 986-998 (2008).

26 Allen JA, Jost JM, Setola V et al. Disovery of $\beta$-arrestin-biased dopamine D2 ligands for probing signal transduction pathways essential for antipsychotic efficacy. Proc. Natl Acad. Sci. USA 108(45), 18488-18493 (2011).

27 Civell O, Nothacker HP, Saito Y et al. Novel neurotransmitters as natural ligands for orphan $\mathrm{G}$ protein-coupled receptors. Trends Neurosci. 24(4), 230-237 (2001).

28 Bräuner-Osborne H, Jensen AA, Sheppard PO et al. Cloning and characterization of a human orphan family $\mathrm{C} G$ protein- coupled receptor GPCR5D. Biochim. Biophys. Acta 1518(3), 237-248 (2001).

29 Beets I, Lindemans M, Janssen T et al. Deorphanizing G protein-coupled receptors by a calcium mobilization assay. Methods Mol. Biol. 789, 377-391 (2011).

30 Bercher M, Hanson B, Van Staden C et al. Agonists of the human orphan G2A receptor identified from inducible G2A expression and beta-lactamase screening. Assay Drug Dev. Technol. 7(2), 133-142 (2009).

31 Johnson EN, Shi X, Cassaday J et al. A 1536-well [(359S] GTPgammaS scintillation proximity assay for ultra-highthroughput screening of an orphan galphai-coupled GPCR. Assay Drug Dev. Technol. 6(3), 327-337 (2008).

32 Dohlman HG. Thematic minireview series: new directions in G protein-coupled receptor pharmacology. J. Biol. Chem. 290, 19469-19470 (2015).

33 Romanova EV, Sweedler JV. Peptidomics for the discovery and characterization of neuropeptides and hormones. Trends Pharmacol. Sci. 36, 579-585 (2015).

34 Gonzalez-Maeso J. GPCR oligomers in pharmacology and signaling. Mol. Brain 4(1), 20 (2011).

35 Rozenfeld R, Gupta A, Gagnidze K et al. AT1R-CBR heteromerization reveals a new mechanism for the pathogenic properties of angiotensin II. EMBO J. 30(12), 2350-2363 (2011).

36 Achour L, Kamal M, Jockers R et al. Using quantitative BRET to assess $\mathrm{G}$ protein-coupled receptor homo- and heterodimerization. Methods Mol. Biol. 756, 183-200 (2011).

37 Cottet M, Albizu L, Comps-Agrar L et al. Time resolved FRET strategy with fluorescent ligands to analyze receptor interactions in native tissues: application to GPCR oligomerization. Methods Mol. Biol. 746, 373-387 (2011).

38 Maurel D, Comps-Agrar L, Brock C et al. Cell-surface protein-protein interaction analysis with time-resolved FRET and snap-tag technologies: applications to $\mathrm{G}$ protein-coupled receptor oligomerization. Methods Mol. Biol. 756, 201-214 (2011).

39 Kumari P, Ghosh E, Shukla AK. Emerging approaches to GPCR ligand screening for drug discovery. Trends Mol. Med. 21, 687-701 (2015).

40 Eberini I, Daniele S, Parravicini C et al. In silico identification of new ligands for GPR17: a promising therapeutic target for neurodegenerative diseases. J. Comput. Aided. Mol. Des. 25(8), 743-752 (2011).

41 Cooke RM, Brown AJ, Marshall FH, Mason JS. Structures of $G$ protein-coupled receptors reveal new opportunities for drug discovery. Drug Discov. Today 20, 1355-1364 (2015).

42 Beuming T, Lenselink B, Pala D, McRobb F, Repasky M, Sherman W. Docking and virtual screening strategies for GPCRs in drug discovery. Methods Mol. Biol. 1335, 251-276 (2015).

43 Jazayeri A, Dias JM, Marshall FM. From G protein-coupled receptor structure resolution to rational drug design. J. Biol. Chem. 290, 19489-19495 (2015).

44 Gentry PR, Sexton PM, Christopoulos A. Novel allosteric modulators of $\mathrm{G}$ protein-coupled receptors. J. Biol. Chem. 290, 19478-19488 (2015). 